\title{
Non-Supersymmetric Open String Vacua
}

\author{
Carlo Angelantonj \\ Centre de Physique Theorique, Ecole Polytechnique, \\ 91128 Palaiseau CEDEX, France \\ E-mail: langelant@cpht.polytechnique.fn
}

ABSTRACT: We review the construction of non-supersymmetric open string vacua in various dimensions. They can be obtained either projecting the (compactified) non-supersymmetric 0B theory, or applying the Scherk-Schwarz mechanism to open strings. Generically, these vacua generate a non-vanishing cosmological constant. However, one can construct particular kinds of Scherk-Schwarz compactifications with vanishing cosmological constant, at least for low orders, based on asymmetric orbifolds. A generic feature of these models is that supersymmetry remains unbroken on the branes at all mass level, while it is broken in the bulk in a way that preserves Fermi-Bose degeneracy at each mass level in the perturbative string spectrum

\section{Introduction}

Motivated by the AdS/CFT correspondence con-

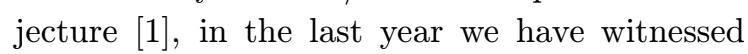
to an increasing interest in non-supersymmetric string theories. On the one hand, the type $0 \mathrm{~B}$ theory has been proposed as the gravitational description (on some appropriate background) of non-supersymmetric gauge theories on D3-branes [2]. On the other hand, the presence of nonsupersymmetric conformal fixed lines has suggested the existence of non-supersymmetric string vacua with vanishing cosmological constant [i3] Since type 0 theories have only bosonic excitations, this last scenario requires non-standard orbifold compactifications of type II superstrings [i4i|i]

Consistent non-supersymmetric vacuum configurations in various dimensions have been recently constructed in different contexts. In the Field Theory limit the Scherk-Schwarz mechanism [5] provides an elegant realization of supersymmetry breaking by compactification. The higher dimensional fields are single valued on the internal manifold up to a (quantized) $R$-symmetry transformation. As a result, the scale of supersymmetry breaking is quantized in units of the inverse size of the internal manifold. This mechanism has been extended to the full perturbative spectrum in models of oriented closed strings [i, 6$]$ and in the corresponding open descendants [1] [].

It has long been known that, besides the five supersymmetric strings, there are a number of non-supersymmetric theories in ten dimensions [8] It was shown in [1] the the $0 \mathrm{~A}$ and $0 \mathrm{~B}$ theories allow the construction of open descendants following [1] $\left.{ }_{1}^{\prime}\right]$. Typically, these theories include in their spectrum tachyonic modes (both in the closed and in the open sector) and thus have often been regarded as toy models. Recently, it has been shown that tachyons in the open sector can be stabilized [1] ${ }_{1}^{1} 1_{1}^{1}$ and then do not represent an inconsistency of the theory. Nevertheless, in order to study consistent string vacua one should get rid of all tachyonic modes. To this end, one can use the crosscap constraint [1 12 in $]$ to change the world-sheet projection, $\Omega$, in the closed unoriented sector and remove all tachyonic modes both in the closed and open unoriented sectors [1] 13

Typically, these two different approaches to the construction of non-supersymmetric string vacua all generate a non-vanishing cosmological constant at one loop. Thus, in order to describe the four-dimensional low-energy world one should find mechanisms that break supersymmetry without generating any cosmological constant at least 
on suitable branes. These kinds of compactifications do exist and are related to asymmetric orbifolds [īi $]$. The main idea consists finding particular kinds of projections that break supersymmetry only in the (anti-)holomorphic sector, thus ensuring the vanishing of the (one-loop) partition function. When projected with respect to the combined action of all the orbifold generators, the resulting model is non-supersymmetric, but has an equal number of fermionic and bosonic degrees of freedom at each mass level.

This paper is organized as follows. In Section 2 we review some basic facts about the construction of open descendants, and in particular discuss the crosscap constraint. In Section 3 we discuss the construction of tachyon-free open descendants of the 0B theory in six and four dimensions. In Section 4 we present non-supersymmetric vacuum configurations of open strings with vanishing cosmological constant. These are open descendants of the model introduced in [1] $\left.\overline{1}_{1}^{\prime}\right]$.

\section{The crosscap constraint}

Let us review some known facts about the construction of open descendants. The starting point is a theory of oriented closed strings invariant under the world-sheet parity $\Omega$. For example, one can consider type IIB or type 0 theories in $D=10$, or any $d$-dimensional theory whose torus partition function

$$
\mathcal{T}=\sum_{a, b} \chi_{a} N_{a b} \bar{\chi}_{b}
$$

is defined through a GSO projection $N_{a b}$ compatible with world-sheet parity. Then the $\Omega$ projection halves the torus amplitude $\mathcal{T}$ and adds to it the contribution of the Klein bottle. Actually, one has the freedom to project onto states invariant under a modified world-sheet symmetry $\Omega^{\prime}=\Omega \mathcal{J}$, where $\mathcal{J}$ is some automorphism of the parent theory. Typically, $\mathcal{J}$ corresponds to a different GSO projection in the parent theory that manifests itself in a different modular invariant combination of characters (the matrix $N_{a b}$ ) in the torus partition function. However, there are cases in which $\mathcal{J}$ reflects an ambiguity in defining the Klein bottle amplitude associated to a given GSO projection of the closed oriented string. In this case, they represent exhotic solutions to the crosscap constraint [i] $\overline{2}$ ].

The action of $\mathcal{J}$ on a given state $|a\rangle$ associated to the character $\chi_{a}$ appearing diagonally in $\mathcal{T}$ translates into a sign $\sigma_{a}$ in the corresponding contribution to the Klein bottle amplitude

$$
\mathcal{K}=\frac{1}{2} \sum_{a} \sigma_{a} K_{a} \chi_{a}
$$

consistently with supersymmetry (if present) and with the crosscap constraint

$$
\sigma_{a} \sigma_{b} \sigma_{c}=+1 \quad \text { if } \quad N_{a b c} \neq 0,
$$

where the tensor $N_{a b c}$ encodes the fusion rules of the states $|a\rangle$.

An interesting example is the compactification of type IIB on a circle of radius $R$. In this case, the $\Omega$-projection is accompained by a $Z_{2}$ shift on the compact coordinate and then the resulting Klein bottle amplitude

$$
\mathcal{K}=\frac{1}{2}\left(V_{8}-S_{8}\right) \sum_{m}(-)^{m} q^{(m / R)^{2}}
$$

introduces minus signs for the odd momentum

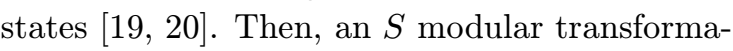
tion reveals that there are no contributions from massless states to the transverse channel that, as a result, does not develop any tadpole for massless unphysical states. One is not allowed to introduce D-branes, and the theory does not contain open strings.

More examples of generalized $\Omega^{\prime}$-projections are offered by the type 0B theory. One has four distinct choices, namely the standard $\Omega$-projection and three additional $\Omega \mathcal{J}$-projections, with $\mathcal{J}=$ $\delta$ (the $Z_{2}$ shift of the previous example), $\mathcal{J}=$ $(-)^{F_{\mathrm{R}}}$ and $\mathcal{J}=(-)^{G_{\mathrm{R}}}$, where $F_{\mathrm{R}}\left(G_{\mathrm{R}}\right)$ is the space-time (world-sheet) fermion number operator. In $D=10$ these different projections have been analyzed in [1 $\left.13^{\prime}\right]$. Particularly interesting is the $\mathcal{J}=(-)^{G_{\mathrm{R}}}$ case, that results in a nonsupersymmetric non-tachyonic model. The nonperturbative consistency of these models has been recently addressed in [21,]. Their conclusion is that the $\Omega(-)^{F_{\mathrm{R}}}$ projection is ruled out since in the decompactification limit it does not lift to any symmetry of M-theory. This leaves only the standard $\Omega$ projection and the non-tachyonic one. 


\section{Open descendants of the $0 B$ string}

The starting point is the $0 \mathrm{~B}$ string in $D=10$. It can be constructed as an orbifold of type IIB, where the symmetry to be gauged is $(-)^{F_{\mathrm{L}}+F_{\mathrm{R}}}$. Obviously, this projection removes all the (spacetime) fermions and introduces, in the twisted sector, a tachyon and additional RR states. In terms of characters of affine $\mathrm{SO}(8)$ at level one, the massless and massive excitations of the $0 \mathrm{~B}$ string can then be collected in the torus partition function

$$
\mathcal{T}=\left|O_{8}\right|^{2}+\left|V_{8}\right|^{2}+\left|S_{8}\right|^{2}+\left|C_{8}\right|^{2},
$$

aside from transverse bosonic modes. Due to the presence of the tachyon, the theory is unstable unless one goes off-criticality, and the vev of the tachyon is chosen to precisely compensate the deficit of central charge [22"]. Ten-dimensional stable non-tachyonic vacuum configurations can indeed be constructed modding out type 0B by the combination $\Omega(-)^{G_{\mathrm{R}}}\left[\overline{1} \overline{3}_{1}\right.$. Since the Klein bottle amplitude now antisymmetrizes all the states associated to the $\mathrm{O}_{8}$ character, this projection removes the tachyon from the closed spectrum. Typically, this is not sufficient to guarantee the stability of the open descendants. A new complex tachyon in the bi-fundamental of a gauge group $\mathrm{U}(N) \otimes \mathrm{U}(32+N)$ appears in the open unoriented sector, where each unitary group comes from the two different D9-branes that exist in type 0B. However, tadpole conditions do not fix the number of D9 and, say, D9'-branes separately. As a result, one is free to choose $N=0$, finally obtaining a non-tachyonic model with fixed gauge group. It should be noted that the consistency of the construction forces one to relax the dilaton tadpole. For 0B theories this does not cause any problem, since NS and R fields are no longer related by space-time supersymmetry. The tadpole of the dilaton, or of any other physical field, can then be disposed by the FishlerSusskind mechanism [ị

One can now try to generalize this construction to $d$-dimensional vacua. Toroidal compactifications can be treated precisely in the same way, since they do not introduce new structures. On the contrary, interesting new features are present in orbifold compactifications. Since the start- ing theory is not supersymmetric, one can study both "supersymmetric" orbifolds [114,, 15i supersymmetric" ones [1] ${ }^{1} \overline{6}^{1}{ }^{1}$. For "supersymmetric" $T^{4} / Z_{N}$ and $T^{6} / Z_{N}$ an exhaustive analysis shows that, for generic $N$, the untwisted sector includes a tachyon, directly related to the ten-dimensional tachyon of $0 \mathrm{~B}$, invariant under the action of $Z_{N}$. Typically, one finds additional (complex) tachyons in the twisted sectors. However, these can not be projected out in the open descendant by any $\Omega \mathcal{J}$ projection at generic (nonrational) points in the moduli space since, for a geometric action of the orbifold generators, the associated partition function belongs to the family of charged conjugate modular invariants. This means that states in the $\theta$-twisted sector are paired with states in the $\theta^{-1}$-twisted one, and the net number of tachyons is then simply halved by any $\Omega^{\prime}$-projection. An exception are the orbifolds $T^{6} / Z_{3}$ and $T^{4} / Z_{2}$, since they do not generate tachyonic modes in the twisted sectors.

The $T^{6} / Z_{3}$ orbifold [1 14 , i 15 , resembles the one studied in [23] aside from the fact that now one is starting from the $0 \mathrm{~B}$ theory. The lowlying excitations of the resulting theory comprise a tachyon, the metric tensor, two abelian vectors and sixty scalars from the untwisted sector and $162=27 \times 6$ scalars from the 27 fixed points in the twisted sectors. The only states that are effectively left-right (anti-)symmetric are the tachyonic vacuum, $g_{\mu \nu}$, the two $A_{\mu}$ and two scalars related to the dimensional reduction of the dilaton and of the (dualized) NS-NS antisymmetric tensor. Then, the standard Klein bottle amplitude would project out the two vectors and the $B_{\mu \nu}$, while keeping the tachyon. On the contrary, combining the world-sheet parity with the world-sheet fermion number, $\Omega(-)^{G_{\mathrm{R}}}$, has the virtue of removing the tachyon from the spectrum while keeping one of the two vectors, consistently with the crosscap constraint. The remaining massless states are simply halved by any $\Omega^{\prime}$-projection, since their holomorphic and antiholomorphic parts come from different sectors. Summarizing, the non tachyonic spectrum com-

\footnotetext{
${ }^{1}$ By "supersymmetric" orbifolds here we mean orbifold compactifications that would give supersymmetric vacua if one started from type II, type I or (supersymmetric) heterotic strings.
} 
prises the graviton, an abelian vector and 111 scalars.

Moving to the open unoriented sector, also in this case we see a doubling of the gauge group. This reflects the doubling of the RR sector in the OB theory, that introduces two different kinds of D9-branes. In addition, the $(-)^{G_{\mathrm{R}}}$ projection enhances the $\mathrm{SO}(n)$ factors that one would naively expect for a $Z_{3}$ orbifold [2] Thus, tadpole cancellations ${ }^{2}$ partially fix the form of the Chan-Paton gauge group

$$
\begin{aligned}
G_{\mathrm{CP}}= & {\left[\mathrm{U}(n) \otimes \mathrm{U}(m)^{2}\right]_{99} \otimes } \\
& \otimes\left[\mathrm{U}(8+n) \otimes \mathrm{U}(12+m)^{2}\right]_{9^{\prime} 9^{\prime}} .
\end{aligned}
$$

while leaving the total dimension arbitrary. As for the ten-dimensional case, one finds in the spectrum a complex tachyon in the $99^{\prime}$ sector. It is then evident that the choice $n=m=0$ leads to an open sector free of tachyons with a $\mathrm{U}(8) \otimes$ $\mathrm{U}(12)^{2}$ gauge group. The remaining (charged) massless excitations comprise three scalars in the $[(\overline{\mathbf{8}}, \mathbf{1 2}, \mathbf{1}) \oplus(\mathbf{8}, \mathbf{1}, \overline{\mathbf{1 2}}) \oplus(\mathbf{1}, \overline{\mathbf{1 2}}, \mathbf{1 2}) \oplus$ c.c $]$ representations, one Dirac fermion in the $(\mathbf{2 8}, \mathbf{1}, \mathbf{1}) \oplus$ $(\mathbf{1}, \mathbf{1 2}, \mathbf{1 2})$ representations, and, finally, three chiral fermions in the $(\overline{\mathbf{8}}, \mathbf{1}, \overline{\mathbf{1 2}}) \oplus(\mathbf{8}, \mathbf{1 2}, \mathbf{1}) \oplus(\mathbf{1}, \overline{\mathbf{6 6}}$,

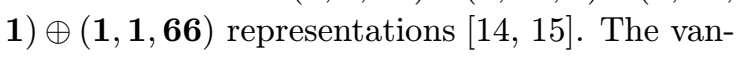
ishing of the irreducible part of the gauge anomaly is a consequence of the cancellation of twisted tadpoles [2] $\left.{ }^{2} \overline{1}\right]$.

Let us now turn to the $T^{4} / Z_{2}$ orbifold. As one would expect, here we have a richer structure, since the orbifold group includes an element that squares to the identity (the generator itself).

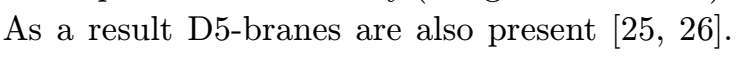
Moreover, the projection $\Omega(-)^{G_{\mathrm{R}}}$ introduces minus signs in the twisted sector of the Klein bottle amplitude, consistently with the fact that the fusion of an untwisted state with a twisted one gives another state that belongs to the twisted sector: the crosscap constraint then forces one to introduce further signs in the twisted sector. As we have already discussed, the inclusion of the world-sheet fermion number in the $\Omega^{\prime}$-projection removes the tachyon from the closed unoriented sector. The massless states that survive the projection give a chiral spectrum consisting of the

\footnotetext{
${ }^{2}$ Also in this case, as in the $T^{4} / Z_{2}$ case that we will discuss later on, the Fishler-Susskind mechanism is to be invoked to cure the uncancelled dilaton tadpole.
}

metric tensor, 4 antiself-dual 2-forms, 20 selfdual 2-forms and 99 scalars, while the open unoriented sector contains two pairs of D9 and D5branes with a Chan-Paton gauge group

$$
\begin{aligned}
G_{\mathrm{CP}}= & {\left[\mathrm{U}(n)_{99} \otimes \mathrm{U}(16+n)_{9^{\prime} 9^{\prime}}\right] \otimes } \\
& \otimes\left[\mathrm{U}(m)_{55} \otimes \mathrm{U}(16+m)_{5^{\prime} 5^{\prime}}\right]
\end{aligned}
$$

The open tachyons correspond to open strings stretched between D9 and D9' branes and D5 and $\mathrm{D} 5^{\prime}$ branes. It is then evident that if one sets $n$ and $m$ to zero, consistently with tadpole conditions, the resulting model is free of any closed and open tachyons. The remaining massless scalars and spinors charged under the Chan-Paton gauge group contribute to the cancellation of the irreducible part of the anomaly polynomial, while a generalized Green-Schwarz mechanism is at work to cancel the residual reducible anomaly $\overline{2} \overline{2} \overline{7}$.

Actually, this model allows two different spectra of charged states $\left[1 \overline{4}^{\prime}, 1 \overline{5}^{\prime}\right]$. This is due to the possibility of adding discrete Wilson lines in the Möbius amplitude, i.e. relative phases between holes and crosscaps ferent $P$ transformation, and, thus, in a different Möbius vacuum amplitude. Precisely the inclusion of discrete Wilson lines in $D=6$ allowed the authors of [9.9] to construct the $\mathrm{U}(16) \otimes \mathrm{U}(16)$ model, rediscovered after some years in [2]-̄i]. Different $T^{4} / Z_{2}$ type $0 \mathrm{~B}$ descendants neatly can be constructed at rational points in the internal lattice. For example, starting from the $\mathrm{SO}(8)$ lattice as in $\left[\overline{9}_{1}^{1}, \overline{2}_{2} 0_{0}^{\prime}\right]$ new massless scalars collapse to zero mass, in a way reminiscent of what happens in the bosonic and heterotic strings, for which one has symmetry enhancement at special points in the moduli space. Moreover, one has several choices of inequivalent sign assignements for the Klein bottle amplitude, that lead to different non-tachyonic vacuum configurations. Due to the presence of an antisymmetric tensor background in the $\mathrm{SO}(8)$ lattice, one expects ChanPaton gauge groups of reduced rank [20 2$]$ and additional self-dual 2-forms related to a different

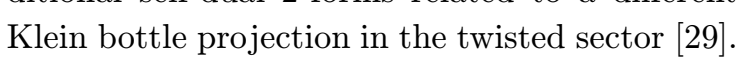

Compactifications on the simplest instance of "non-supersymmetric" orbifolds have been recently considered by Blumenhagen and Kumar [1] 
internal coordinates are reversed under the $Z_{2}$. Typically, this particular projection does not satisfy the constraints of modular invariance for the superstring. It acts as a $Z_{4}$ action on the Ramond sector, and introduces phases in the NS$\mathrm{R}$ and R-NS sectors. However, the action on the type $\mathrm{OB}$ is perfectly consistent. One can project further the theory by $\Omega(-)^{G_{\mathrm{R}}}$ to get nontachyonic four-dimensional open string vacua. The result is similar to the $6 \mathrm{D}$ one previously discussed, but with D3-branes instead of D5-branes.

\section{Non-supersymmetric vacua with vanishing cosmological constant}

Non-supersymmetric vacua with a vanishing cosmological constant can be obtained as asymmetric orbifolds of type II and/or type I superstrings. The simplest instance of these models can be obtained considering the generators [1] $\left.{ }^{1}{ }^{\prime}\right]$

$$
\begin{aligned}
& f=\left[\left(-1^{4}, 1 ; 1^{5}\right),\left(0^{4}, v_{\mathrm{L}} ; \delta^{4}, v_{\mathrm{R}}\right),(-)^{F_{\mathrm{R}}}\right] \\
& g=\left[\left(1^{5} ;-1^{4}, 1\right),\left(\delta^{4}, w_{\mathrm{L}} ; 0^{4}, w_{\mathrm{R}}\right),(-)^{F_{\mathrm{L}}}\right],(
\end{aligned}
$$

that define a non-abelian space group orbifold $S$ [30i]. Here the first entry inside the square brackets denotes rotations, the second denotes shifts on the internal compactification lattice while the third entry denotes projections on states with even left (right) space-time fermion number. A semicolon separates holomorphic and antiholomorphic coordinates. The asymmetric nature of the orbifold requires that the internal fourdimensional lattice splits into a product of four circles with self-dual radius $R=\sqrt{\alpha^{\prime}}$. Level matching requires $\delta$ be a shift by $R / 2$, while no further constraints are imposed on the radius of the fifth coordinate, for which the shifts $v_{\mathrm{L}, \mathrm{R}}=w_{\mathrm{R}, \mathrm{L}}$ act as $A_{2}$ shifts in the notation of [3in].

Due to the presence of $(-)^{F_{\mathrm{L}, \mathrm{R}}}$, the $g(f)$ generator projects out all gravitini coming from the (anti)holomorphic sector, and therefore the combined action of $f$ and $g$ breaks supersymmetry completely, while ensuring the vanishing of the one-loop contribution to the cosmological constant. In [32i] it was argued that higher order perturbative corrections to the cosmological constant vanish as well. There are, however, non-perturbative contributions originating from wrapped D-branes that can be studied perturbatively on the dual heterotic model [1] $\left.{ }^{1}\right]$ or computing the D-brane spectra using boundary-state techniques [33i].

In order to construct the orbifold (4.11), it is simpler to restrict onself to the abelian point group $\bar{P}[30 \overline{0}]$, thus defining a new compactification lattice $\Gamma_{4,4}(\mathrm{SO}(8)) \oplus \Gamma_{1,1}(R)$. In this case, $\bar{P}$ reduces to an abelian $Z_{2} \otimes Z_{2}$ orbifold. The resulting one-loop partition function involves only the modular orbit generated by the untwisted sector. This fact has two different interpretations in the space group and in the point group approaches to the orbifold. In the former, it is due to the fact that the path integral receives contributions only from commuting spin structures [30"] , whereas in the latter the disconnected orbit vanishes due to the simultaneous action of shifts and rotations [34].

Invariance under world-sheet parity forces one to start with the type IIB superstring modded

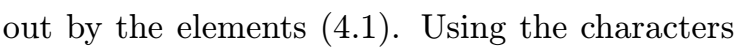
of affine $\mathrm{SO}(2 n)$ at level one, the massless contributions to the torus amplitude read

$$
\begin{aligned}
\mathcal{T}_{\text {untw }} \sim & \left|V_{4} O_{4}\right|^{2}+\left|S_{4} S_{4}\right|^{2}+ \\
& -\left(O_{4} V_{4}\right)\left(\bar{C}_{4} \bar{C}_{4}\right)-\left(C_{4} C_{4}\right)\left(\bar{O}_{4} \bar{V}_{4}\right), \\
\mathcal{T}_{\text {f } g-\text { tw }} \sim & 8\left|O_{4} S_{4}-C_{4} O_{4}\right|^{2},
\end{aligned}
$$

that translate into the following five-dimensional field content: the metric tensor, seven abelian vectors, six scalars and eight fermions from the untwisted sector, and eight vectors, forty scalars and sixteen fermions from the $f g$-twisted sector. Due to the presence of the shifts, the $f$ and $g$ twisted sectors are massive, so that no massless gravitini originate from them.

The construction of the open descendants requires suitable contributions from the Klein bottle, annulus and Möbius strip world-sheet topologies $\left[{ }_{1}^{1} 0_{n}^{1}, \bar{g}\right]$. In the following we will just describe the main features of the model and we refer the interested reader to [35in for details ${ }^{3}$. At the massless level one finds the following contribu-

\footnotetext{
${ }^{3}$ See also [36i] for a different approach to the construction of the D-brane structure for the asymmetric orbifold $(4.1)$.
} 
tions:

$$
\mathcal{K} \sim\left(V_{4} O_{4}-S_{4} S_{4}\right)+(6-2)\left(O_{4} S_{4}-C_{4} O_{4}\right)
$$

from the Klein bottle amplitude,

$$
\begin{aligned}
\mathcal{A} \sim & 2 M \bar{M}\left(V_{4} O_{4}-S_{4} S_{4}\right)+ \\
& +\left(M^{2}+\bar{M}^{2}\right)\left(O_{4} V_{4}-C_{4} C_{4}\right)
\end{aligned}
$$

from the annulus amplitude, and, finally,

$$
\mathcal{M} \sim-(M+\bar{M})\left(\hat{O}_{4} \hat{V}_{4}-\hat{C}_{4} \hat{C}_{4}\right)
$$

from the Möbius amplitude. Since the action of the generators $f$ and $g$ is equivalent to a Tduality transformation, the open unoriented sector involves only one kind of charges, a linear combination of (wrapped) D9 and D5-branes. In the Klein bottle amplitude we have explicitly written how the $\Omega$-projection acts on the $f g$-twisted sector. Not all the contributions are treated in the same way. $\Omega$ (anti-)symmetrizes the (R-R) NS-NS sector of six ( $f$-invariant combinations of) fixed points, and (anti-)symmetrizes the (NSNS) R-R sector of the remaining two fixed points. This is due to the presence of a non-vanishing $B_{a b}$ background in the $\mathrm{SO}(8)$ lattice $\left[\overline{3} \overline{7}_{1}^{1} \overline{2} \overline{9} \overline{1}\right]$.

The cancellation of tadpoles of unphysical massless states in the transverse channel then fixes the size of the Chan-Paton gauge group, so that $M=\bar{M}=8$. The rank reduction is due both to the presence of a non-vanishing background for the NS-NS antisymmetric tensor in the $\mathrm{SO}(8)$ lattice $\left[\overline{2} \overline{8}, \overline{3} \overline{7}_{\mathrm{b}}^{\prime}, \overline{2} \overline{2}_{1}^{\prime}\right]$ and to the identification of (wrapped) D9 and D5-branes under the action of the orbifold generators (4.1). The massless closed unoriented spectrum comprises a graviton, 4 abelian vectors, 31 scalars and 12 fermions. The massless unoriented open sector
In conclusion, one can study the limiting behavior of the type I model for large and small radius. For $R \rightarrow 0$ new uncancelled tadpoles arise in the transverse channels, thus inducing linear divergences in the five-dimensional gauge theory

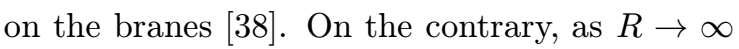
the action of the $f$ and $g$ generators is trivialized, while the sector projected and/or twisted by the combined $f g$ action survives. The decompactification limit then corresponds to a $T^{4} / Z_{2}$ orbifold. Due to the presence of a non-vanishing flux for the NS-NS $B_{a b}$, the type I compactification comprises at massless level additional tensor multiplets and has a Chan-Paton group with reduced rank. One finds a $D=6 \mathcal{N}=(1,0)$ supergravity multiplet coupled to 5 tensor multiplets and 16 hypermultiplets from the closed unoriented sector together with a vector multiplet in the adjoint representation of $\mathrm{U}(8) \otimes \mathrm{U}(8)$ and charged hypermultiplets in the representations $(\mathbf{2 8} \oplus \overline{\mathbf{2 8}}, \mathbf{1}) \oplus(\mathbf{1}, \mathbf{2 8} \oplus \overline{\mathbf{2 8}}) \oplus(\mathbf{8}, \overline{\mathbf{8}}) \oplus(\overline{\mathbf{8}}, \mathbf{8})$ from the open unoriented sector, one of the models of $\left[\bar{y}_{1}, \overline{2} \overline{0}\right]$. The presence of additional tensor multiplets calls for a generalized Green-Schwarz mechanism for the cancellation of the residual anomaly [품] .

\section{Acknowledgments}

It is a pleasure to thank I. Antoniadis and K. Förger for a pleasant and stimulating collaboration. I would also like to thank A. Sagnotti for stimulating discussions. Research supported in part by the EEC TMR contract ERBFMRXCT96-0090.

\section{References}

11] J. Maldacena, Adv Theor Math Phys, 2 (1998) $23 \overline{1}$

[2] I.R. Klebanov and A.A. Tseytlin, 'Nucl. Phys. B'

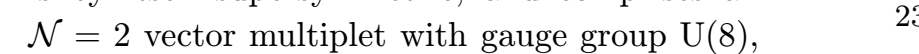

, _as_well_as_a bypermultiplet in the_representations

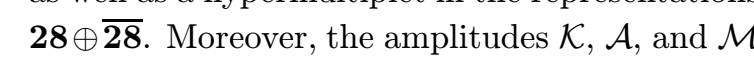

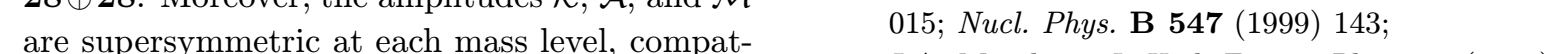


- ibty with the- vanishing of the (onre=loop) ecosmro= - - . - .

- fogicat constant. 'This has to be- contrasted with what usually happens in 'M-theory breaking' of type I models [i] $]$ and in the heterotic dual of $[1,18,135]$, where supersymmetry is realized in the "gauge sector" only at the massless level.
A. Armoni and B. Kol, 'Non-supersymmetric large $N$ gauge theories from type 0 brane configurations', hep-th/9906081;

S. Seki, 'Baryon configurations in the UV and IR regions of type 0 string theory', 
hep-th/9906210;

I.R. Klebanov, 'Tachyon stabilization in the

' - . . Ads/CFT correspondence', 'hep-th/9906220'.

[3] S. Kachru and E. Silverstein, 'Phys. Rev. Lett.' - 80 (1998) 4855

[4] S. Kachru, J. Kumar and E. Silverstein, Pי Phys.' - Rev. D $\mathbf{5 9}$ (1999) 106004.

[5] J. Scherk and J.H. Schwarz, №ch. Phys. B 153, : -

[6] R. Rohm, 'Nucl. Phys. B 237 (1984) 553; C. Kounnas and M. Porrati, 'Nucl. Phys. B 310! - -

S. Ferrara, C. Kounnas, M. Porrati and F. Zwirner, 'Nucl. Phys. B 318 (1989) 75í;

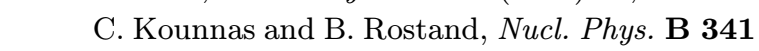

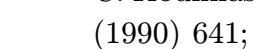
I. Antoniadis and C. Kounnas, 'Phys. Lett. B'

-

E. Kiritsis and C. Kounnas, 'Nucl. Phys. B $\mathbf{5 0 3}$

$=2=(1997) 117$

[7] I. Antoniadis, E. Dudas and A. Sagnotti, 'ívucl.'.' p. Phys. B $\mathbf{5 4 4}(1999) 469$

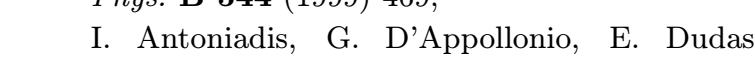
and A. Sagnotti, 'Partial breaking of supersymmetry, open strings and M-theory', hep-th/9812118.

[8] L.J. Dixon and J.A. Harvey, iNucl. Phys. B $\mathbf{2} \overline{\mathbf{7}}$ I

N. Seiberg and E. Witten, iNucl. Phys. B $\mathbf{2} 7 \mathbf{7}^{-1}$ (1986) 272

L. Alvarez-Gaumé, P. Ginsparg, G. Moore and

C. Vafa, 'Phys. Lett. B 171 (1986) 155.'.

- -[9]- AF. Bianchir antc A- Sagnotti, $P$ Pltys. Eett. $\mathbf{B} \mathbf{2 4 7}$ : - [10]- A. -Sagnotti, in- Cargèse -87, -Non-perturbative

' - - - Qurantum fielel-Theory, -G.-Mack; et al. -(Eels.), Pergamon Press, Oxford, 1988, p. 521.

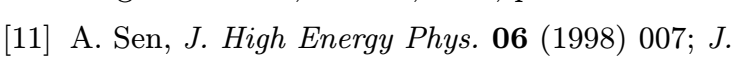
High Energy Phys. 08 (1998) 010 J. High En」 ergy Phys. 08 (1998) 012 - 09 -1998) 023; $\bar{J}_{-}$High Energy Phys 10_(1998)

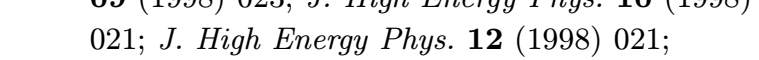
O. Bergman and M.R. Gaberdiel, Phys. Lett. B

[ - 441 (1998) 133; 013

E. Witten,

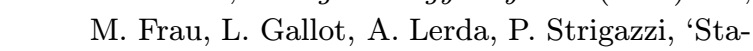

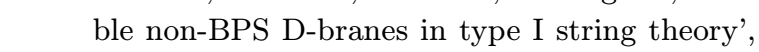
- - - -

[12]_D. Fioravanti, G. Pradisi and A. Sagnotti, Phys.í [ - _ett. B_321 (1994) $349_{i}^{\prime}$
G. Pradisi, A. Sagnotti and Ya.S. Stanev, iPhys.1 Lett. B 354 (1995) 279; , 'Phys. Lett. B _ 356! (1995) 230; 'Phys. Lett. B 381_(1996)_97?

[13] A. Sagnotti, 'Some surprises of open-string theories', hep-th/9509080'; 'Surprises in open-string perturbation theory', hep-th/9702093'

[14] C. Angelantonj, Phys. Lett. B

[15] R. Blumenhagen, A. Font and D. Lüst, 'Tachyon-free orientifolds of type 0B strings in various dimensions', hep-th/9904069",

[16] R. Blumehagen and A. Kumar, 'A note on orientifolds and dualities of type 0B string theory', hep-th/9906234.

[17]_ W. Fischler and L. Susskind, Phys. Lett. B.171' (1986) 383;' 'Phys. Lett. B_173 (1986)_262'

[18] J.A. Harvey, iPhys. Rev. D $\mathbf{5 9}(1999) 26002$.

[19]_A. Dabholkar and J. Park, iNucl. Phys. B $\mathbf{4} \overline{7} \overline{1}$ $(\overline{19} \overline{6}) \overline{70} \overline{1}$.

[20] C. Angelantonj, M. Bianchi, G. Pradisi, A. Sagnotti and Ya.S. Stanev, Phys. Lett. B $\mathbf{3 8}$, $(1996) 743$

[21] O. Bergman and M.R. Gaberdiel, 'Dualities of type 0 strings', hep-th/9906055!

[22] G. Ferretti and D. Martelli, 'On the construction of gauge theories from noncritical type 0 strings', hep-th/9811208;

G. Ferretti, J. Kalkkinen and D. Martelli, 'Noncritical type 0 string theories and their field theory duals', hep-th/9904013!

[23] C. Angelantonj, M. Bianchi, G. Pradisi, A. Sagnotti and Ya.S. Stanev, 'Phys. Lett. B $\mathbf{3} 8 \mathbf{5}_{1}^{\prime}$ $(1996)$ 96.

[24] J. Polchinski and Y. Cai, 'Nucl. Phys. B 296i $(1988)$ 91:

G. Aldazabal, D. Badagnani, L.E. Ibáñez and A.M. Uranga, 'Tadpole versus anomaly cancellation in $D=4, D=6$ compact IIB orientifolds', hep-th/9904071'

[25] G. Pradisi and A. Sagnotti, 'Phys. Lett. B 216! $(1 \overline{9} 89)-59_{r}^{\prime}$

[26] E. Gimon and J. Polchinski, 'Phys. Rev. D $(\overline{19} 96)-\overline{1} 6 \overline{6} 7$.

[27] A. Sagnotti, 'Phys. Lett. B 294 (1992) 196; S. Ferrara, R. Minasian and A. Sagnotti, iNucl.' Phys. B 474 (1996) $323^{\prime}$

S. Ferrara, F. Riccioni and A. Sagnotti, iNucl.'

F. Riccioni and A. Sagnotti, 'Phys. Lett. B 436' (1998) 298. 
[28] M. Bianchi, G. Pradisi and A. Sagnotti, 'Nucl.'.' : - Phys. B $\mathbf{3 7 6}(1992) 365$

M. Bianchi, iNucl. Phys. B 528 (1998) 73;

E. Witten, H. High Energy Phys. 02 (1998)_006.

[29] C. Angelantonj, in preparation.

[30] L. Dixon, J.A. Harvery, C. Vafa and E. Witten, 'Nucl. Phys. B 261_(1985)_678;', 'Nucl. Phys. B- - 274

[31] C. Vafa and E. Witten, 'Nucl. Phys. $\mathbf{4 6}$ (Proc.' ' - Suppl.) (1996) 225!

[32] S. Kachru and E. Silverstein, J. High Energy Phys. 11 (1998) 001' 'J. High Energy Phys. 01' (1999) 004 .

[33] B. Körs, 'D-brane spectra of nonsupersymmetric, asymmetric orbifolds and nonperturbative contributions to the cosmological constant', hep-th/9907007.

[34] E. Kiritsis and C. Kounnas, 'Nucl. Phys. B $\mathbf{5 0 3}$ (1997) 117.

[35] C. Angelantonj, I. Antoniadis, K. Förger, 'Nonsupersymmetric type I strings with zero vacuum energy, 'hep-th/9904092,

[36] R. Blumenhagen and L. Görlich, 'Nucl. Phys. B. - - 5

[37] Z. Kakushadze, G. Shiu and S.-H.H. Tye, í : _ Rev. D $\mathbf{5 8}$ (1998) 086001!

[38] I. Antoniadis and C. Bachas, Phys. Lett. B 450; $(1999)$ 83i 